\title{
Equilibria in Altruistic Economic Growth Models
}

\author{
Łukasz Balbus $^{1}$ (D) Anna Jaśkiewicz ${ }^{2}$ (D) Andrzej S. Nowak ${ }^{1}$ \\ Published online: 2 March 2019 \\ (c) The Author(s) 2019
}

\begin{abstract}
In this paper, we consider a stochastic economic growth model in the form of an intergenerational dynamic game. Both paternalistic and non-paternalistic components are present in the model. Under very general assumptions allowing for unbounded utility functions and weakly continuous transitions, we establish the existence of Markov perfect equilibria that consist of a consumption strategy and an indirect utility function. In the pure paternalistic case, we obtain new results on equilibria. An important point of our contribution is that we make no separability assumptions on the utility functions of generations.
\end{abstract}

Keywords Stochastic game - Intergenerational game · Altruistic growth models · Markov perfect equilibrium

\section{Introduction}

The literature concerning various aspects of altruism in economic models is pretty large. For a comprehensive survey with historical notes, the reader is referred to $[9,21,33,35]$ and their references. This paper is devoted to study some mathematical issues related to existence of equilibria in a large class of economic growth models with altruism between generations. Intergenerational altruism has usually been modeled in two ways. In the paternalistic model, the utility of the current generation depends on its own consumption and the consumptions of other generations. In other words, the generation cares about what all or some successors will consume, but it does not take into account the utilities the successors derive from the act of consumption. In the non-paternalistic model, each generation derives utility from its own consumption and the utilities of future generations.

Łukasz Balbus

1.balbus@wmie.uz.zgora.pl

Anna Jaśkiewicz

anna.jaskiewicz@pwr.edu.pl

Andrzej S. Nowak

a.nowak@wmie.uz.zgora.pl

1 Faculty of Mathematics, Computer Science and Econometrics, University of Zielona Góra, Podgórna 50, 65-246 Zielona Góra, Poland

2 Faculty of Pure and Applied Mathematics, Wrocław University of Science and Technology, 50-370 Wrocław, Poland 
First strong results on the existence of Markov perfect equilibria in paternalistic economic growth models with deterministic transitions were established by Bernheim and Ray [11] and Leininger [28]. They assume that each generation cares only about consumption of its immediate successor. From the mathematical point of view, their proofs are rather complicated. A simpler and more direct method was used by Balbus et al. [3]. Certain extensions of the works of $[11,28]$ to models with specific stochastic production functions are surveyed in $[8,9,25]$. However, more general results the reader may find in $[4,5]$, where the transition probability function obeys a natural weak continuity condition. Such transition probabilities are extensively used in economics, since economic dynamics are typically described by some difference equations with additive or multiplicative shocks. The most general paternalistic model was considered by Balbus et al. [5]. However, their results concern non-atomic transition probabilities. The model studied in [4] allows to cover both deterministic production functions and the stochastic transitions that satisfy a stochastic dominance assumption. The drawback of this approach lies in the compactness of the state space and in the separability of the utility function which takes into account only an immediate descendant for each generation.

In contrast to the paternalistic case, there have been very few rigorous studies of models assuming the non-paternalistic altruism. As suggested by Ray [35], "this framework appears to be of somewhat greater interest in the context of applications." Examples include the works of [10,29]. For instance, Ray [35] described a general model with deterministic transitions involving non-paternalistic altruism and formulated an equilibrium concept, but its existence remains an open problem. Balbus et al. [6] showed that an equilibrium in a stochastic version of Ray's framework exists provided that the transition probabilities are non-atomic. The other group of models were considered by Barro [10] or Loury [29], who dealt with only one descendant for each generation. However, the existence of an equilibrium consisting of an indirect utility and an optimal consumption (or saving) strategy in the aforementioned models can be studied by dynamic programming methods using contraction mapping theorems. It is worth mentioning that these methods were also applied to the wide class of various decision processes with recursive utilities, see for instance $[17,18]$.

In this paper, we study a version of so-called mixed models with both paternalistic and non-paternalistic components. A need for studying mixed models is expressed on page 113 in [35]. An approach to mixed models (two-sided altruism) is given by Hori [23]. He considers a rather specific model with pretty strong assumptions on the utility and deterministic transition functions. An equilibrium is shown to exist in this model by the Schauder fixed-point theorem. Although the approach of [23] concerns some specific model, it is inspiring for us.

In our model, we assume that every generation considers only its immediate successor. The equilibrium problem studied in this paper is a double fixed-point problem. One fixed point is obtained for an indirect utility function via a contraction mapping with a nonlinear discount function. This is a sort of recursive utility extensively discussed in economics, see Becker and Boyd [15]. The second one (in an appropriate strategy space) corresponds to Nash equilibrium in an intergenerational game. Our basic tool is the Schauder-Tychonoff fixed-point theorem (see e.g., Dugundji and Granas [16]). We would like to emphasize that the indirect utility that we consider depends on both consumption and endowment of the generation. This approach is generalized in the model of [35]. A similar mixed model is studied in the recent paper of [2]. However, he considers risk-sensitive generations (uses different utility functions), makes much stronger assumptions on the stochastic transition function and proves existence of equilibria in the class of Borel measurable randomized strategies. Therefore, his approach is essentially different from the one applied in this paper. Our proofs are based on techniques used in $[3,4]$ with some necessary modifications. We prove a general existence theorem for 
the mixed model with unbounded utility functions by applying the weighted norm approach, which was well developed in dynamic programming (see Wessels [40]; Jaśkiewicz et al. [27]). However, instead of standard exponential discounting, we use a nonlinear discount function. As a by-product, we considerably extend our earlier result for paternalistic models given in [4]. The most important point is that no separability condition on the utility function is imposed.

The rest of the paper is organized as follows. Section 2 contains preliminaries. Section 3 derives the utility function which incorporates the paternalistic and non-paternalistic altruism and formulates an equilibrium. Section 4 presents basic assumptions and existence theorems. Examples satisfying our conditions are given in Sect. 5. Finally, the proofs are collected in Sects. 6 and 7.

\section{Preliminaries}

In this section, we introduce some notation and state a few auxiliary results. As usual, $\mathbb{R}$ stands for the set of all real numbers and $\mathbb{N}$ is the set of all positive integers. Let $S=\mathbb{R}_{+}=[0, \infty)$, $S_{+}=\mathbb{R}_{+} \backslash\{0\}$ and $A(s):=[0, s], s \in S$.

Let $X$ be the vector space of all continuous from the left functions $\phi: S \rightarrow \mathbb{R}$ such that $\phi(0)=0$ and that the restriction of $\phi$ to any bounded interval $[0, m](m \in \mathbb{N})$ has a bounded variation. We assume that $X$ is endowed with the topology of weak convergence. Recall that a sequence $\left(\phi_{n}\right)$ converges weakly to $\phi \in X$ if and only if $\phi_{n}(s) \rightarrow \phi(s)$ as $n \rightarrow \infty$ at any continuity point $s \in S$ of $\phi$. Here, we point out that $s=0$ is considered as a continuity point of $\phi \in X$ if $\lim _{s \rightarrow 0^{+}} \phi(s)=\phi(0)$. The weak convergence of $\left(\phi_{n}\right)$ to $\phi$ is denoted by $\phi_{n} \stackrel{*}{\rightarrow} \phi$.

Let $F$ be the set of all continuous function from the left mappings $c: S \rightarrow S$ such that the function $y(s):=s-c(s)$ is non-decreasing and $c(s) \in A(s)$ for all $s \in S$. Note that $s \rightarrow y(s)$ is lower semicontinuous. Thus, $c \in F$ is upper semicontinuous. Define

$$
I:=\{y \in X: y(s)=s-c(s) \text { where } s \in S, c \in F\} .
$$

Observe that $I \subset X$. Moreover, $s=0$ is the continuity point of every function in $F$ or $I$.

Lemma $1 X$ is a locally convex topological vector space. The sets $I$ and $F$ are convex and sequentially compact in $X$.

For a more detailed discussion, consult Lemma 1 and Appendix in [7].

In the sequel, we shall use a generalized version of the contraction mapping principle due to [31], see also Theorem 5.2 in [16].

Proposition 1 Let $(Y, \rho)$ be a complete metric space and $\delta: S \rightarrow S$ be a continuous function such that $\delta(0)=0$ and $\delta(r)<r$ for all $r \in S_{+}$. Assume that $T: Y \rightarrow Y$ is a mapping such that

$$
\rho(T x, T y) \leq \delta(\rho(x, y)) \text { for all } x, y \in Y,
$$

and define $T^{1} x:=T x, T^{m+1} x:=T T^{m} x, x \in Y, m \in \mathbb{N}$. Then, $T$ has a unique fixed point $x^{*} \in Y$ and $\lim _{n \rightarrow \infty} \rho\left(T^{n} x_{0}, x^{*}\right)=0$ for any $x_{0} \in Y$.

We shall also assume that $z \rightarrow \delta(z) / z$ is non-increasing on $S_{+}$. This assumption implies that $\delta$ is subadditive and hence

$$
\left|\delta\left(z_{1}\right)-\delta\left(z_{2}\right)\right| \leq \delta\left(\left|z_{1}-z_{2}\right|\right) \text { for all } z_{1}, z_{2} \in S .
$$


Moreover, the fact that $z \rightarrow \delta(z) / z$ is non-increasing implies that $\delta(\kappa z) / \kappa z \leq \delta(z) / z$ for any $z \in S_{+}$and $\kappa \geq 1$. Hence, it holds

$$
\delta(\kappa z) \leq \kappa \delta(z) \text { for all } \kappa \geq 1, z \in S .
$$

As in the dynamic programming literature (see Jaśkiewicz et al. [27]), we call $\delta$ a discount function.

Let $d>0$ and $\eta:[d, \infty) \rightarrow \mathbb{R}$ be a fixed function. Following Milgrom and Shannon [32], we say that $\eta$ has the strict single crossing property on $[d, \infty)$, when the following holds: if there exists some $x \geq d$ such that $\eta(x) \geq 0$, then for each $x^{\prime}>x$, we have $\eta\left(x^{\prime}\right)>0$. It is worth to note that $\eta$ need not be increasing, see Example 3 in [3].

Let $u: S \times S \rightarrow \mathbb{R}_{+}$be a function of the form $u(a, w)=g\left(u_{o}(a, w)\right)$. We make the following assumptions.

(U1) $g$ is continuous, increasing, and $g(0)=0$.

(U2) $u_{o}(0,0)=0$ and $u_{o}$ is continuous on $S \times S$ and increasing in each variable.

(U3) For any $w_{2}>w_{1}$ in $S, l>0$ and for each $d>0$, the function $D_{l} u_{o}(x):=u_{o}\left(x, w_{2}\right)-$ $u_{o}\left(x+l, w_{1}\right)$ has the strict single crossing property on $[d,+\infty)$.

Remark 1 The form of $u(a, w)=g\left(u_{o}(a, w)\right)$ is very convenient for verification of our assumptions. From them, it follows that $u$ is nonnegative. Using this representation, one can immediately see that (U1)-(U3) hold for $u(a, w)$ such as $\ln (1+\sqrt{a}+w), \sqrt{1+\sqrt{a}+w}-1$, $\sqrt{\sqrt{a}+w}$ or $\ln (1+w \sqrt{a})$. The function $u$ plays the role of "aggregator" in our definitions of the utility functions in altruistic growth models. Note that the aggregator of [20] of the form $u(a, w)=\left((1-\beta) a^{\sigma}+\beta w^{\sigma}\right)^{\frac{1}{\sigma}}$ with $\beta, \sigma \in(0,1)$ also satisfies (U1)-(U3).

The study of unbounded from below utilities $u$ requires new methods and seem to be difficult to handle in this setup.

Remark 2 Bernheim and Ray [11] in their study of an altruistic growth model did not assume that $u$ is the composition of $u_{o}$ and $g$. They directly imposed conditions on $u: u$ is strictly concave in its first argument and $u$ satisfies the increasing difference property (ID). (ID) says that for each $w_{1}>w_{2}$ in $S$, the function $x \rightarrow u\left(x, w_{1}\right)-u\left(x, w_{2}\right)$ is non-decreasing. The function $u$ that satisfies (ID) is called supermodular. Supermodular functions turn out to be useful in operations research and game theory, see Topkis [38,39]. Balbus et al. [3], on the other hand, assumed that $u$ meets the strict single crossing property. On page 517 in [3], it is also shown that if $u$ satisfies (ID) and is strictly concave in the first argument, then $u$ possesses the strict single crossing property. Observe that the functions $\ln (1+\sqrt{a}+w)$, $\sqrt{1+\sqrt{a}+w}-1, \sqrt{\sqrt{a}+w}$ do not satisfy (ID). However, they are compositions of an increasing function $g$ with $u_{o}$ satisfying (ID). Below, we give an example of $u=g \circ u_{o}$ where $g$ is increasing and $u_{o}$ satisfies condition (U3) and does not have the (ID) property. This example also illustrates how (U3) can be checked directly using the definition of $u_{o}$.

Example 1 Let $u(a, w):=(w+\ln (a+w))^{\sigma}$ where $\sigma \in(0,1)$. Clearly, $g(z)=z^{\sigma}$ is increasing and continuous and $u_{o}(a, w)=w+\ln (a+w)$. The function $u_{o}$ does not satisfy (ID), since $\frac{\partial^{2} u_{o}}{\partial a \partial w}<0$. For any $w_{2}>w_{1}$ in $S, l>0$ we have

$$
D_{l} u_{o}(x):=u_{o}\left(x, w_{2}\right)-u_{o}\left(x+l, w_{1}\right)=w_{2}-w_{1}+\ln \left(\frac{1+w_{2}+x}{1+w_{1}+l+x}\right) .
$$

If $w_{2} \geq w_{1}+l$, then $D_{l} u_{o}(x)>0$ for all $x \geq d$, where $d$ is an arbitrary number larger than 0 . If $w_{2}<w_{1}+l$, then $D_{l} u_{o}(x)$ is increasing and there is a unique point $x_{o} \geq d$ such that $D_{l} u_{o}\left(x_{o}\right)=0$. Thus, $D_{l} u_{o}$ has the strict single crossing property on $[d,+\infty)$. 


\section{Markov Perfect Equilibria in Altruistic Growth Economies}

Consider an infinite sequence of generations labeled by $t \in T=\mathbb{N}$. There is one commodity, which may be consumed or invested. Every generation lives one period and in the paternalistic case derives utility from its own consumption and consumption of its immediate descendant. In the non-paternalistic case generation, $t \in T$ takes into account a utility for consumption of generation $t+1$. In this paper, we are interested in mixed model where both paternalistic and non-paternalistic components are present. Generation $t \in T$ receives the endowment $s_{t} \in S$ and chooses consumption level $a_{t} \in A\left(s_{t}\right)=\left[0, s_{t}\right]$. The investment $i_{t}:=s_{t}-a_{t}$ determines the endowment of its successor according to some transition probability $q$ from $S$ to $S$, which depends on $i_{t} \in A\left(s_{t}\right)$. Let $\Phi$ be the set of Borel measurable functions $\phi: S \rightarrow S$ such that $\phi(s) \in A(s)$ for each $s \in S$. A strategy or policy for generation $t \in T$ is a Borel measurable function $c_{t}: S \rightarrow S$ such that $c_{t}\left(s_{t}\right) \in A\left(s_{t}\right)$ for all $s_{t} \in S$. The set of all strategies for each generation is denoted by $\Pi$.

Let $v: S \rightarrow \mathbb{R}_{+}$be a continuous increasing function such that $v(0)=0$. Assume that generation $t \in T$ consumes $a \in A\left(s_{t}\right)$ in state $s_{t}=s$ and the following generation is going to use a strategy $c_{t+1}=c^{\prime} \in \Pi$. Then, the term

$$
\mathcal{E}_{i} v\left(c^{\prime}\right):=\int_{S} v\left(c^{\prime}\left(s^{\prime}\right)\right) q\left(d s^{\prime} \mid i\right)
$$

is a generation $t$ 's evaluation of consumption policy $c^{\prime}$ of generation $t+1$ under investment $i=s-a$. Let $U\left(c^{\prime}\right)\left(s^{\prime}\right)$ denote the (Borel measurable in $s^{\prime}$ ) utility for generation $t+1$ resulting from its consumption policy $c^{\prime}$ in state $s^{\prime} \in S$. This utility can also be evaluated by generation $t$ under investment $i=s-a$ by computing the expected value with respect to the probability measure $q(\cdot \mid i)$. More formally, generation $t$ can consider

$$
\mathcal{E}_{i} U\left(c^{\prime}\right):=\int_{S} U\left(c^{\prime}\right)\left(s^{\prime}\right) q\left(d s^{\prime} \mid i\right)
$$

Assume that $\mathcal{E}_{i} v\left(c^{\prime}\right)$ and $\mathcal{E}_{i} U\left(c^{\prime}\right)$ are aggregated with the aid of the function $W: \mathbb{R}_{+} \times \mathbb{R}_{+} \rightarrow$ $\mathbb{R}_{+}$, i.e., $w=W\left(\mathcal{E}_{i} v\left(c^{\prime}\right), \mathcal{E}_{i} U\left(c^{\prime}\right)\right)$ is calculated. Then, the aggregated utility for generation $t$ is obtained by aggregating $a \in A(s)$ and $w$ by the function $u$ discussed in Preliminaries. More precisely, the utility of generation $t$ under investment $i=s-a$ is defined as

$$
P\left(a, c^{\prime}, U\left(c^{\prime}\right)\right)(s):=u\left(a, W\left(\mathcal{E}_{s-a} v\left(c^{\prime}\right), \mathcal{E}_{s-a} U\left(c^{\prime}\right)\right)\right) .
$$

Similarly as in [35] or [29], we can call $U$ an indirect utility for generation $t \in T$. However, one should note that indirect utilities in their approaches are functions depending on endowments only.

If $s=s_{t}, a=c_{t}\left(s_{t}\right), c^{\prime}=c_{t+1}, i_{t}=s_{t}-c_{t}\left(s_{t}\right)$, this utility equals

$$
P\left(c_{t}\left(s_{t}\right), c_{t+1}, U\left(c_{t+1}\right)\right)\left(s_{t}\right)=u\left(c_{t}\left(s_{t}\right), W\left(\mathcal{E}_{i_{t}} v\left(c_{t+1}\right), \mathcal{E}_{i_{t}} U\left(c_{t+1}\right)\right)\right) .
$$

This clearly shows that the utility of generation $t$ depends on its own consumption in state $s_{t}$, the expectation of its own evaluation $v$ of consumption of generation $t+1$ (paternalistic altruism component) and the utility $U$ of consumption of generation $t+1$ (non-paternalistic altruism component). In the sequel, we impose additional assumptions on functions $v$ and $W$ and the transition probability $q$ to cover an unbounded case.

Definition 1 A pair $\left(c^{*}, U^{*}\right)$ with $c^{*} \in \Pi$ is a stationary Markov perfect equilibrium $(S M P E)$ with the utility defined in (2), if

$$
U^{*}\left(c^{*}\right)(s)=P\left(c^{*}(s), c^{*}, U^{*}\left(c^{*}\right)\right)(s)=\sup _{a \in A(s)} P\left(a, c^{*}, U^{*}\left(c^{*}\right)\right)(s) \text { for every } s \in S .(4)
$$


Note that in (4), we deal with a double fixed-point problem. The strategy $c^{*}$ is the best response for every generation $t$, if its immediate successor is going to use $c^{*}$, and each generation evaluates its consumption strategy $c^{*}$ using the same function $U^{*}$. Following Ray [35], one can say that it is assumed in Definition 1 that "there exist an indirect utility function and a consumption strategy (policy), both depending on current endowment, such that each generation finds it optimal to adopt that consumption strategy, provided its immediate descendant uses the same policy and exhibit the given indirect utility. Moreover, the indirect utility function generated by the generations maximization problem is also the same as that announced by its descendant."

In the pure paternalistic case, the utility for generation $t$ is of simpler form

$$
\tilde{P}\left(c_{t}\left(s_{t}\right), c_{t+1}\right)\left(s_{t}\right)=u\left(c_{t}\left(s_{t}\right), \mathcal{E}_{i_{t}} v\left(c_{t+1}\right)\right) .
$$

The analogous form to (2) is

$$
\tilde{P}\left(a, c^{\prime}\right)(s):=u\left(a, \mathcal{E}_{s-a} v\left(c^{\prime}\right)\right),
$$

where $a \in A(s)$ is a consumption of generation $t, i=s-a$ is its investment in state $s \in S$ and $c^{\prime}$ is a consumption strategy of generation $t+1$.

The definition of equilibrium is similar to that given in [11,28,34] or [3,4].

Definition 2 A strategy $c^{*} \in \Pi$ is a stationary Markov perfect equilibrium (SMPE) with the utility defined in (5), if

$$
\tilde{P}\left(c^{*}(s), c^{*}\right)(s)=\sup _{a \in A(s)} \tilde{P}\left(a, c^{*}\right)(s) \text { for every } s \in S .
$$

Remark 3 By putting $i=s-a$ in (2) and (5), we can rewrite Eqs. (4) and (6) in a more convenient forms for our proofs. Namely, observe that

$$
P\left(c^{*}(s), c^{*}, U^{*}\left(c^{*}\right)\right)(s)=\sup _{i \in A(s)} u\left(s-i, W\left(\mathcal{E}_{i} v\left(c^{*}\right), \mathcal{E}_{i} U^{*}\left(c^{\prime}\right)\right)\right) .
$$

and

$$
\tilde{P}\left(c^{*}(s), c^{*}\right)(s)=\sup _{i \in A(s)} u\left(s-i, \mathcal{E}_{i} v\left(c^{*}\right)\right) .
$$

Remark 4 If $W(b, r)=r$, then the utility in (3) reduces to a pure non-paternalistic case, which is of the form

$$
R\left(c_{t}\left(s_{t}\right), U\left(c_{t+1}\right)\right)\left(s_{t}\right):=u\left(c_{t}\left(s_{t}\right), \mathcal{E}_{i_{t}} U\left(c_{t+1}\right)\right) .
$$

This model was studied by Loury [29] with a deterministic transition function and by Ray [35], who considered countably many descendants for each generation. An equilibrium existence in the model of [35] is still an open problem. In the stochastic case with non-atomic transition, the existence of such equilibrium was established by Balbus et al. [6].

Moreover, we would like to point out that in this pure non-paternalistic case with utility (7), the fixed point $U^{*}$ is independent of $c \in F$. This follows from the fact that the fixed point belongs to the subspace of functions considered here. The consumption strategy $c^{*} \in F$ in Definition 1, on the other hand, can be found by dynamic programming technique, see Loury [29], Durán [17,18]. Our results within this special framework can be considered as an extension of the results of [18] to models with nonlinear discount function.

In the general case, $c^{*}$ from Definitions 1 and 2 is a solution to a non-cooperative game problem. This is a symmetric Nash equilibrium in a game played by generations. Further comments on this issue, the reader may find in [25]. 


\section{Basic Assumptions and Main Results}

Let $\operatorname{Pr}(S)$ be the set of all probability measures on the state space $S$. We recall that a sequence $\left(\mu_{n}\right)$ of probability measures on $S$ converges weakly to some $\mu_{0} \in \operatorname{Pr}(S)\left(\mu_{n} \Rightarrow \mu_{0}\right.$ in short) if, for any bounded continuous function $h: S \rightarrow \mathbb{R}$, it holds that

$$
\lim _{n \rightarrow \infty} \int_{S} h(s) \mu_{n}(d s)=\int_{S} h(s) \mu_{0}(d s) .
$$

We already made three assumptions (U1)-(U3) on the aggregator $u$. Below, we provide additional conditions on the primitive data that will be imposed in our two main results. To include unbounded from above utilities, we shall apply a weighted norm approach inspired by the papers in dynamic programming (see Wessels [40]; Hernández-Lerma and Lasserre [22]; Jaśkiewicz and Nowak [24]) or recursive utility theory (see Boyd [14]; Durán [17,18]).

Let $\omega: S \rightarrow[1, \infty)$ be a continuous non-decreasing function. Further, $\omega$ will be called a weight function. We now make some basic assumptions on the transition probability.

(Q1) Assume that $\lambda_{j}: S \rightarrow[0,1], j \in J:=\{1, \ldots, N\}$, are continuous functions such that $\sum_{j=1}^{N} \lambda_{j}(i)=1$ for all $i \in S$. In addition, suppose that there exist transition probabilities $q_{j}$ from $S$ to $S, j \in J$, such that for each $i \in S$, we have

$$
q(\cdot \mid i)=\sum_{j=1}^{N} \lambda_{j}(i) q_{j}(\cdot \mid i) .
$$

Moreover, for every $j \in J, q_{j}(\{0\} \mid 0)=1$, and the transition probability $q_{j}(\cdot \mid i)$ has the Feller property, i.e., if $i_{n} \rightarrow i_{0}$ in $S$ as $n \rightarrow \infty$, then $q\left(\cdot \mid i_{n}\right) \Rightarrow q\left(\cdot \mid i_{0}\right)$.

(Q2) Every transition probability $q_{j}(\cdot \mid y)$ in (8) satisfies the stochastic dominance condition, i.e., if $z \rightarrow Q_{j}(z \mid i):=q_{j}([0, z] \mid i)$ is the cumulative distribution function for $q_{j}(\cdot \mid i)$, then for any $i_{1}<i_{2}$ and $z \in S$, we have that $Q_{j}\left(z \mid i_{1}\right) \geq Q_{j}\left(z \mid i_{2}\right)$.

(Q3) For every $z \in S$, the set $S^{z}:=\{i \in S: q(\{z\} \mid i)>0\}$ is countable.

(Q4) The function $i \rightarrow \int_{S} \omega\left(s^{\prime}\right) q\left(d s^{\prime} \mid i\right)$ is continuous on $S$.

(Q5) We have

$$
\kappa_{0}:=\sup _{s \in S} \sup _{a \in A(s)} \frac{\int_{S} \omega\left(s^{\prime}\right) q\left(d s^{\prime} \mid s-a\right)}{\omega(s)}<\infty .
$$

As in preliminaries, we make the following assumption on the discount function $\delta$.

(D) $\delta: S \rightarrow S$ is continuous and non-decreasing, and $\delta(z)<z$ for all $z \in S_{+}$. (Hence, it follows that $\delta(0)=0$.) Moreover, the function $z \rightarrow \delta(z) / z$ defined on $S_{+}$is nonincreasing.

We can now continue our assumptions on the utility function.

(U4) The function $v: S \rightarrow \mathbb{R}_{+}$is increasing and continuous, and $v(0)=0$.

(U5) If $v$ is unbounded, then the function $i \rightarrow \int_{S} v\left(s^{\prime}\right) q\left(d s^{\prime} \mid i\right)$ is continuous on $S$.

(U6) The function $W: \mathbb{R}_{+} \times \mathbb{R}_{+} \rightarrow \mathbb{R}_{+}$is continuous and increasing in each variable, and $W(0,0)=0$.

(U7) There exists a constant $\kappa_{1}>0$ such that for each $s \in S$, we have

$$
u\left(s, W\left(\|v\|_{\omega} \kappa_{0} \omega(s), 0\right) \leq \kappa_{1} \omega(s) .\right.
$$

Here, $\|v\|_{\omega}$ is defined as $\sup _{x \in S} v(x) / \omega(x)$ and is assumed to be finite. 
(U8) For any $r_{1}, r_{2} \in \mathbb{R}_{+}$and every $s \in S, a \in A(s), b \geq 0$, we have

$$
\left|u\left(a, W\left(b, r_{1}\right)\right)-u\left(a, W\left(b, r_{2}\right)\right)\right| \leq \delta\left(\left|r_{1}-r_{2}\right|\right) .
$$

For any $f: F \times S \rightarrow \mathbb{R}$ we define

$$
\|f\|_{\omega}:=\sup _{s \in S} \sup _{c \in F} \frac{|f(c, s)|}{\omega(s)} .
$$

Let $C(F \times S)$ be the Banach space of all continuous functions $f: F \times S \rightarrow \mathbb{R}$ such that $\|f\|_{\omega}<\infty$. Further, in some cases we shall write $f(c)(s)$ instead of $f(c, s)$ for any $f \in C(F \times S)$.

We can now state our main results.

Theorem 1 Let assumptions (Q1)-(Q5), (U1)-(U4), (U6)-(U8) and (D) be satisfied. Assume also that if $\kappa_{0}>1$, then $\delta\left(\kappa_{0} r\right)<r$ for all $r>0$. Then, there exists an SMPE $\left(c^{*}, U^{*}\right)$ in the sense of Definition 1 and $c^{*} \in F, U^{*} \in C(F \times S)$.

Remark 5 It should be noted that $U^{*}$ in Theorem 1 is unique. However, in a general case the uniqueness of $c^{*}$ is not guaranteed. This issue for similar models is also considered in [8,30].

In the paternalistic case, we can drop the assumptions involving the discount function $\delta$ and weight function $\omega$.

Theorem 2 Under assumptions (Q1)-(Q3) and (U1)-(U5), there exists an SMPE $c^{*}$ in the sense of Definition 2 and $c^{*} \in F$.

Remark 6 (a) Assumptions (Q1)-(Q5) were used to study stochastic games of resource extraction by Jaśkiewicz and Nowak [26]. (Q3) was first used in [4], and it holds for the purely deterministic transitions and as well their convex combinations. Moreover, it may occur that all sets $S^{z}$ in (Q3) are empty. This means that $q$ is non-atomic. Other possible transitions are presented in Sect. 5. Here, we only wish to point out that (Q3) is not satisfied, for instance, for $q$ of the form

$$
q(\cdot \mid i)=(1-i) \tilde{\delta}_{\{0\}}(\cdot)+i q_{u}(\cdot \mid i),
$$

where $\tilde{\delta}_{\{0\}}(\cdot)$ is the Dirac measure at point 0 and $q_{u}(\cdot \mid i)$ is the uniform distribution on $S:=[0,1]$. Then, $q(\{0\} \mid i)=1-i$ and $q(\{0\} \mid i)>0$ for all $i \in[0,1)$. Hence, the cardinality of $S^{0}$ is continuum.

(b) Although the transition probability $q$ has the Feller property, it need not satisfy the stochastic dominance condition, even if the expected new state $\int_{S} s^{\prime} q\left(d s^{\prime} \mid i\right)$ is increasing with respect to the investment $i$ (consult with Examples 4-6 in [26]).

(c) Assumption (D) was used in dynamic programming models studied in [27].

Remark 7 The classes of strategies $F$ and $I$ were used to study bequest equilibria by Bernheim and Ray [11] and Leininger [28]. Their proofs are rather complicated. A simpler method was proposed in [3,4]. Moreover, this class of semicontinuous strategies is also useful in dynamic game models of resource extraction, see Sundaram [37], Dutta and Sundaram [19], Jaśkiewicz and Nowak [26].

Remark 8 Theorem 1 is new, even in the pure non-paternalistic case (see Remark 4). Theorem 2, on the other hand, considerably extends the work of [4] (in the risk-neutral case), where the state space $S$ is a compact interval and thus, the utility function $u$ is bounded. More importantly, Balbus et al. [4] assume that $\tilde{P}(a, c)(s)=\hat{u}(a)+\int_{S} v\left(c\left(s^{\prime}\right)\right) q\left(d s^{\prime} \mid s-a\right)$, i.e., the utility is separable. Hence, these results generalize ones of [4] in the two aforementioned directions. We wish to emphasize that certain techniques and ideas used in $[3,4]$ are useful in our setup, but after a suitable adaptation. 


\section{Examples}

In this section, we give four examples. The first example is inspired by a two-sided altruism model of [23]. The remaining three examples provide some specific functions in the altruistic growth economies for which the imposed conditions are satisfied. Examples 3 and 4 refer to the assumptions used in Theorem 1, whereas Example 5 illustrates conditions used in Theorem 2.

Example 2 Assume that each generation $t \in T$ consists of two populations: old and young members. The young population in generation $t$ becomes old in generation $t+1$. The utility functions for consumptions are: $u_{1}$ for the young population and $u_{2}$ for the old population. Supposing that the members of generation $t$ cooperate, we can maximize $u_{1}\left(a_{t}^{1}\right)+u_{2}\left(a_{t}^{2}\right)$ subject to $a_{t}^{1}+a_{t}^{2}=a_{t}$, where $a_{t} \in A\left(s_{t}\right)$ is the total consumption of generation $t$ in state $s_{t} \in S$. Assuming that the functions $u_{1}$ and $u_{2}$ are increasing and strictly concave, one can show that there exist continuous increasing functions $\psi_{1}$ and $\psi_{2}$ on $S$ such that

$$
\hat{u}\left(a_{t}\right)=\max _{a_{t}^{1}+a_{t}^{2}=a_{t}}\left(u_{1}\left(a_{t}^{1}\right)+u_{2}\left(a_{t}^{t}\right)\right)=u_{1}\left(\psi_{1}\left(a_{t}\right)\right)+u_{2}\left(\psi_{2}\left(a_{t}\right)\right) .
$$

By Lemma 1 in [23], $\hat{u}$ is strictly concave and increasing.

Let $c_{t+1}$ be a consumption strategy of generation $t+1$ and $U\left(c_{t+1}\right)\left(s_{t+1}\right)$ be a chosen (announced) utility function by generation $t+1$ depending on both $c_{t+1}$ and $s_{t+1}$. Then, the aggregated utility for generation $t$ is

$$
\begin{aligned}
& \hat{u}\left(a_{t}\right)+(1-\beta) \int_{S} u_{2}\left(\psi_{2}\left(c_{t+1}\left(s_{t+1}\right)\right)\right) q\left(d s_{t+1} \mid s_{t}-a_{t}\right) \\
& \quad+\beta \int_{S} U\left(c_{t+1}\right)\left(s_{t+1}\right) q\left(d s_{t+1} \mid s_{t}-a_{t}\right),
\end{aligned}
$$

where $\beta \in(0,1)$. Thus, the utility of generation $t$ is the sum of the utility resulting from cooperation of populations in period $t$ and the weighted sum of the utility from consumption of old in period $t+1$ (who were young in period $t$ ) and the expected utility $U$ announced by generation $t+1$ calculated for the strategy $c_{t+1}$ and endowment $s_{t+1}$.

Example 3 Let the transition probability be of the following form

$$
q(\cdot \mid i)=\lambda(i) \tilde{\delta}_{\{i\}}(\cdot)+(1-\lambda(i)) q_{u}(\cdot \mid i),
$$

where $\lambda: S \rightarrow(0,1)$ is a continuous function such that $\lambda(i) \rightarrow 0$ as $i \rightarrow 0$, and $\tilde{\delta}_{\{i\}}(\cdot)$ is the Dirac measure concentrated at point $i$ and $q_{u}(\cdot \mid i)$ is the uniform distribution on [0,2i] with $i \in S$. Obviously, $q_{u}(\{0\} \mid 0)=1$. Note that (Q1)-(Q3) immediately hold. Moreover, the set $S^{z}$ in (Q3) has at most 1 element. Now we prove (Q4) and (Q5) for $\omega(s)=1+s$. Observe that the function

$$
i \rightarrow \int_{S} \omega\left(s^{\prime}\right) q\left(d s^{\prime} \mid i\right)=1+\lambda(i) i+(1-\lambda(i)) i=1+i
$$

is continuous on $S$ and additionally,

$$
\sup _{s \in S} \sup _{i \in A(s)} \frac{\int_{S} \omega\left(s^{\prime}\right) q\left(d s^{\prime} \mid i\right)}{\omega(s)}=\sup _{s \in S} \sup _{i \in A(s)} \frac{1+i}{s+1}=1 .
$$

Hence, we have $\kappa_{0}=1$. Furthermore, we define the function $\delta$ satisfying (D) as

$$
\delta(z)=\alpha z+(1-\alpha) \ln (1+z) \quad \text { with } \alpha \in(0,1), z \in S,
$$


and the function in (U4) as $v(s)=2 \sqrt{s}, s \in S$. Clearly, $\|v\|_{\omega}=1$. Finally, we put $W(b, r)=\delta(b+r),(b, r) \in S \times S$ and $u(a, w)=\ln (1+\sqrt{a}+w),(a, w) \in S \times S$ (see Remark 1). Then, we have

$$
u(a, W(b, r))=\ln (1+\sqrt{a}+\alpha(b+r)+(1-\alpha) \ln (1+b+r)) .
$$

Condition (U8) follows from the mean value theorem, because

$$
\begin{aligned}
\left|u\left(a, W\left(b, r_{1}\right)\right)-u\left(a, W\left(b, r_{2}\right)\right)\right| & =\left|\ln \left(1+\sqrt{a}+\delta\left(b+r_{1}\right)\right)-\ln \left(1+\sqrt{a}+\delta\left(b+r_{2}\right)\right)\right| \\
& \leq\left|\delta\left(b+r_{1}\right)-\delta\left(b+r_{2}\right)\right| \leq \delta\left(\left|r_{1}-r_{2}\right|\right) .
\end{aligned}
$$

The last inequality is due to this fact

$$
\begin{aligned}
\left|\delta\left(b+r_{1}\right)-\delta\left(b+r_{2}\right)\right| & \leq \alpha\left|r_{1}-r_{2}\right|+(1-\alpha)\left|\ln \left(\frac{1+b+r_{1}+r_{2}-r_{2}}{1+b+r_{2}}\right)\right| \\
& \leq \alpha\left|r_{1}-r_{2}\right|+(1-\alpha) \ln \left(1+\frac{\left|r_{1}-r_{2}\right|}{1+b+r_{2}}\right) \\
& \leq \alpha\left|r_{1}-r_{2}\right|+(1-\alpha) \ln \left(1+\left|r_{1}-r_{2}\right|\right)=\delta\left(\left|r_{1}-r_{2}\right|\right) .
\end{aligned}
$$

It remains to check condition (U7) and find the constant $\kappa_{1}$. For every $s \in S$ we obtain

$$
\begin{aligned}
u\left(s, W\left(\|v\|_{\omega} \kappa_{0} \omega(s), 0\right)\right. & =\ln (1+\sqrt{s}+\delta(\omega(s))) \\
& =\ln (1+\sqrt{s}+\alpha \omega(s)+(1-\alpha) \ln (1+\omega(s))) \\
& \leq \ln (1+\sqrt{s}+\omega(s)) \leq \sqrt{s}+1+s \leq \frac{3}{2}(s+1) .
\end{aligned}
$$

Thus, it suffices to take $\kappa_{1}=3 / 2$.

Example 4 Assume that the transition function is deterministic, i.e., $q(\cdot \mid i)=\tilde{\delta}_{\{i+\sqrt[4]{3 i}\}}(\cdot)$. Let the function $W$ be as in Example 3, $\omega(s)=s+l$ for $s \in S$ and some $l \geq 1$ (which will be chosen below). Furthermore, suppose that

$$
\delta(z)=(1-2 \alpha) z+\alpha \ln (1+z) \quad \text { with } \alpha \in(0,1 / 2), z \in S .
$$

Note that $\delta$ obeys all requirements in (D). Conditions (Q1)-(Q4) hold and (Q5) is satisfied with

$$
\kappa_{0}=\sup _{s \in S} \frac{l+s+\sqrt[4]{3 s}}{l+s}=1+\frac{3}{4 \sqrt[4]{l^{3}}} .
$$

This last equality follows from the fact that the function $s \rightarrow 1+\frac{\sqrt[4]{3 s}}{l+s}$ attains its maximum at $s=l / 3$. We now observe that

$$
\delta\left(\kappa_{0} z\right)=(1-2 \alpha) \kappa_{0} z+\alpha \ln \left(1+\kappa_{0} z\right) \leq(1-\alpha) \kappa_{0} z<z
$$

holds if $(1-\alpha) \kappa_{0}<1$. Hence, for every $\alpha \in(0,1 / 2)$, we may choose $l \geq 1$ such that $(1-\alpha) \kappa_{0}<1$ and consequently $\delta\left(\kappa_{0} z\right)<z$ for all $z>0$. Let $u(a, w)=2 \sqrt{1+\sqrt{a}+w}-2$, $(a, w) \in S \times S$ (consult with Remark 1). We have

$$
u(a, W(b, r))=2 \sqrt{1+\sqrt{a}+(1-2 \alpha)(b+r)+\alpha \ln (1+b+r)}-2 .
$$

Assumption (U8) holds, since

$$
\begin{aligned}
\left|u\left(a, W\left(b, r_{1}\right)\right)-u\left(a, W\left(b, r_{2}\right)\right)\right| & =2\left|\sqrt{1+\sqrt{a}+\delta\left(b+r_{1}\right)}-\sqrt{1+\sqrt{a}+\delta\left(b+r_{2}\right)}\right| \\
& \leq \delta\left(\left|r_{1}-r_{2}\right|\right)
\end{aligned}
$$


where the last inequality can be shown in the same manner as in (9). Let us put $v(s)=$ $\ln (1+s), s \in S$, and observe that

$$
\|v\|_{\omega}=\sup _{s \in S} \frac{\ln (1+s)}{s+l} \leq \sup _{s \in S} \frac{\ln (1+s)}{1+s}=\frac{1}{e} .
$$

Finally, we have to prove (U7). Choosing $l>1$ such that $(1-\alpha) \kappa_{0}<1$, we can assure that $\kappa_{0}<e / 2$. Then,

$$
\begin{aligned}
u\left(s, W\left(\|v\|_{\omega} \kappa_{0} \omega(s), 0\right)\right. & =\leq u(s, W(0.5 \omega(s), 0) \\
& =2(\sqrt{1+\sqrt{s}+(1-2 \alpha) 0.5 \omega(s)+\alpha \ln (1+0.5 \omega(s))}-1) \\
& \leq 2(\sqrt{1+\sqrt{s}+(1-2 \alpha) 0.5 \omega(s)+\alpha 0.5 \omega(s)}-1) \\
& \leq 2(\sqrt[4]{s}+\sqrt{0.5(1-\alpha) \omega(s)}) \leq 2(\sqrt[4]{s}+\sqrt{0.5 \omega(s)})<3 \omega(s) .
\end{aligned}
$$

Thus, it suffices to take $\kappa_{1}=3$.

Example 5 In this example, we assume that the next state evolves according to the following recursive equation $s_{t+1}=i_{t}(1+\epsilon) \zeta_{t}$, where $\epsilon>0$ is a fixed interest rate and $\left(\zeta_{t}\right)$ is a sequence of i.i.d. random shocks with values in $[0, \infty)$ and with a distribution $\pi$ such that

$$
\int_{S} \sqrt{\zeta} \pi(d \zeta)<\infty
$$

Thus,

$$
q(B \mid i)=\int_{S} 1_{B}(i(1+\epsilon) \zeta) \pi(d \zeta) \text { for every Borel set } B \subset S, i \in S .
$$

Note that (Q1)-(Q3) are satisfied, if 0 is not an atom of $\pi$. In the paternalistic model, we may define $u(a, w)=(1 / 4 \sqrt{a}+3 / 4 \sqrt{w})^{2}$ for $(a, w) \in S \times S$ and $v(s)=\sqrt{s}$ for $s \in S$. Note that the function

$$
i \rightarrow \int_{S} v\left(s^{\prime}\right) q\left(d s^{\prime} \mid i\right)=\int_{S} v(i(1+\epsilon) \zeta) \pi(d \zeta)=\sqrt{i(1+\epsilon)} \int_{S} \sqrt{\zeta} \pi(d \zeta)
$$

is continuous. Hence, all assumptions in Theorem 2 are satisfied.

\section{Basic Monotonicity Result}

In this section, we provide a useful result that may have applications to various models in optimization. Let $\xi: S \rightarrow S$ be an upper semicontinuous function. For any $s \in S$, define

$$
A_{o}^{\xi}(s):=\arg \max _{i \in A(s)} u(s-i, \xi(i)) \text { and } a_{o}^{\xi}(s):=\min A_{o}^{\xi}(s) .
$$

Under our assumptions (U1)-(U2), the function $i \rightarrow u(s-i, \xi(i))$ is upper semicontinuous on $A(s)$. Therefore, the set $A_{o}^{\xi}(s)$ is non-empty and compact. Thus, $a_{o}^{\xi}(s)$ is well defined. The following result is related to Proposition 1 in [3].

Proposition 2 If (U1)-(U3) are satisfied and $\xi$ is an upper semicontinuous function, then

(a) any selection of the set-valued mapping $s \rightarrow A_{o}^{\xi}(s)$, i.e., a function $\varphi: S \rightarrow S$ such that $\varphi(s) \in A_{o}^{\xi}(s)$ for all $s \in S$ is non-decreasing. 
(b) If, in addition, $\xi$ is continuous from the left, then the function $\phi=a_{o}^{\xi}$ is continuous from the left at any point $s \in S$.

Proof Observe that since $g$ is continuous and increasing, we conclude from (10) that

$$
A_{o}^{\xi}(s)=\hat{A}_{o}^{\xi}(s):=\arg \max _{i \in A(s)} u_{o}(s-i, \xi(i)) .
$$

Therefore, $a_{o}^{\xi}(s):=\min A_{o}^{\xi}(s)=\min \hat{A}_{o}^{\xi}(s)$ and in the remaining part of the proof, we can work with $u_{o}$.

(a) First note that $\varphi(0)=0 \leq \varphi(s)$ for each $s \in S$. Assume that there exist $s_{1}, s_{2} \in S$ such that $0<s_{1}<s_{2}$ and $\varphi\left(s_{1}\right)>\varphi\left(s_{2}\right)$. Let $S_{1}=\left[s_{1}, \infty\right)$ and $A_{1}=\left[0, \varphi\left(s_{1}\right)\right]$. Note that $\varphi\left(s_{1}\right)>0$. Choose any $i_{1}, i_{2} \in A_{1}$ such that $i_{1}>i_{2}$ and define

$$
\eta(s)=u_{o}\left(s-i_{1}, \xi\left(i_{1}\right)\right)-u_{o}\left(s-i_{2}, \xi\left(i_{2}\right)\right), s \in S_{1} .
$$

If $\xi\left(i_{1}\right) \leq \xi\left(i_{2}\right)$, then under our monotonicity assumptions on $u_{o}$, we have $\eta(s)<0$ for all $s \in S_{1}$, and $\eta$ has the strict single crossing property. If $\xi\left(i_{1}\right)>\xi\left(i_{2}\right)$, then we write

$$
\eta(s)=u_{o}\left(s-i_{1}, \xi\left(i_{1}\right)\right)-u_{o}\left(s-i_{1}+\left(i_{1}-i_{2}\right), \xi\left(i_{2}\right)\right), s \in S_{1} .
$$

By (U3), this function has a strict single crossing property. Let $\varphi^{\prime}$ be any function defined on $S_{1}$ such that $\varphi^{\prime}(s) \in \arg \max _{i \in A_{1}} u_{o}(s-i, \xi(i))$ for all $s \in S_{1}$ and $\varphi^{\prime}\left(s_{1}\right)=\varphi\left(s_{1}\right)$ and $\varphi^{\prime}\left(s_{2}\right)=\varphi\left(s_{2}\right)$. By Theorem 4' in [32], $\varphi^{\prime}$ is non-decreasing. Hence, it follows that $\varphi\left(s_{1}\right) \leq \varphi\left(s_{2}\right)$. We have come to a contradiction, which finishes the proof of part $(a)$.

(b) Let $s_{0}>0$. Assume that $s_{n} \uparrow s_{0}$ as $n \rightarrow \infty$. Choose any $i \in\left[0, s_{0}\right)$. Then, we get

$$
u_{o}\left(s_{n}-\phi\left(s_{n}\right), \xi\left(\phi\left(s_{n}\right)\right)\right)=\max _{i^{\prime} \in A\left(s_{n}\right)} u_{o}\left(s_{n}-i^{\prime}, \xi\left(i^{\prime}\right)\right) \geq u_{o}\left(s_{n}-i, \xi(i)\right)
$$

for all but finitely many $n \in \mathbb{N}$. Since $\phi$ is non-decreasing, the limit $i_{0}:=\lim _{n \rightarrow \infty} \phi\left(s_{n}\right)$ exists and $i_{0} \in A\left(s_{0}\right)$. By assumption, the function $\xi$ is continuous from the left. Thus, we have

$$
u_{o}\left(s_{0}-i_{0}, \xi\left(i_{0}\right)\right) \geq u_{o}\left(s_{0}-i, \xi(i)\right), \quad i \in\left[0, s_{0}\right) .
$$

Since $\xi$ is continuous from the left, (11) also holds for $i=s_{0}$. Hence, $i_{0} \in A_{o}^{\xi}\left(s_{0}\right)$. Clearly, $i_{0} \geq a_{o}^{\xi}\left(s_{0}\right)$. If $i_{0}>a_{o}^{\xi}\left(s_{0}\right)=\phi\left(s_{0}\right)$, then there exists some $n \in \mathbb{N}$ such that $s_{n}<s_{0}$ and $\phi\left(s_{n}\right)>\phi\left(s_{0}\right)$. This inequality contradicts the fact that $\phi$ is non-decreasing.

The following auxiliary result is a simple modification of Lemma 3 in [4].

Lemma 2 Let $\varphi \in I$ be any function such that $\varphi(s) \in A_{o}^{\xi}(s)$ for all $s \in \mathcal{D}$ and $\mathcal{D}$ be a dense subset of $S$. If $s \in \mathcal{D}$ is a continuity point of $\varphi$, then the set $A_{o}^{\xi}(s)$ is a singleton.

\section{Proofs of Theorems 1 and 2}

In this section, we assume that assumptions used in Theorems 1 and 2 are satisfied, although they are not explicitly recalled.

Lemma 3 Let $f \in C(F \times S)$. Then, the function $(c, i) \rightarrow \int_{S} f\left(c, s^{\prime}\right) q\left(d s^{\prime} \mid i\right)$ is continuous on $F \times S$. 
Proof First note that both functions $f$ and $f^{\omega}$ defined by $f^{\omega}(c, s):=\omega(s)\|f\|_{\omega}-f(c, s)$ are continuous and nonnegative. A simple adaptation of the proof of Proposition 7.31 in [12] yields that the functions $(c, i) \rightarrow \int_{S} f\left(c, s^{\prime}\right) q\left(d s^{\prime} \mid i\right)$ and $(c, i) \rightarrow \int_{S} f^{\omega}\left(c, s^{\prime}\right) q\left(d s^{\prime} \mid i\right)$ are lower semicontinuous. Since $i \rightarrow \int_{S} \omega\left(s^{\prime}\right) q\left(d s^{\prime} \mid i\right)$ is continuous, it follows that $(c, i) \rightarrow$ $\int_{S} f\left(c, s^{\prime}\right) q\left(d s^{\prime} \mid i\right)$ is simultaneously lower and upper semicontinuous.

Lemma 4 For any $c \in F$, the function $i \rightarrow \int_{S} v\left(c\left(s^{\prime}\right)\right) q\left(d s^{\prime} \mid i\right)$ is upper semicontinuous.

Proof Since $v$ is continuous and increasing and $c \in F$, the function $v(c(\cdot))$ is upper semicontinuous. Hence, $\omega(s)\|v\|_{\omega}-v(c(s))$ is nonnegative and lower semicontinuous. By Proposition 7.31 in [12], the function $i \rightarrow \int_{S}\left(\omega\left(s^{\prime}\right)\|v\|_{\omega}-v\left(c\left(s^{\prime}\right)\right)\right) q\left(d s^{\prime} \mid i\right)$ is lower semicontinuous. Since $\rightarrow \int_{S} \omega\left(s^{\prime}\right) q\left(d s^{\prime} \mid i\right)$ is continuous, the assertion follows.

Lemma 5 For any $c \in F$, the function $i \rightarrow \int_{S} v\left(c\left(s^{\prime}\right)\right) q\left(d s^{\prime} \mid i\right)$ is continuous from the left.

Proof Consider any $q_{j}$ from the representation $(\mathrm{Q} 1)$ of $q$. Then, recall that $q_{j}$ has the Feller property and the stochastic dominance property. Using the Skorohod representation theorem for weak convergence of probability measures (see Billingsley [13]), continuity from the left of $v(c(\cdot))$ and the dominated convergence theorem as in the proof of Lemma 6 in [4], one can conclude that the function $i \rightarrow \int_{S} v\left(c\left(s^{\prime}\right)\right) q_{j}\left(d s^{\prime} \mid i\right)$ is continuous from the left. Since all functions $\lambda_{j}$ in the representation of $q$ in (Q1) are continuous, it follows the assertion of our lemma.

Let $\left(h_{n}\right)$ be a sequence of Borel measurable real-valued functions on $S$. For each $s \in S$, define

$$
\liminf _{n \rightarrow \infty, s^{\prime} \rightarrow s} h_{n}\left(s^{\prime}\right):=\inf \left\{\liminf _{n \rightarrow \infty} h_{n}\left(s_{n}\right): s_{n} \rightarrow s\right\}
$$

and

$$
\limsup _{n \rightarrow \infty, s^{\prime} \rightarrow s} h_{n}\left(s^{\prime}\right):=\sup \left\{\limsup _{n \rightarrow \infty} h_{n}\left(s_{n}\right): s_{n} \rightarrow s\right\}
$$

Lemma 6 Assume that $\mu_{m} \Rightarrow \mu_{0}$ and $\int_{S} \omega(s) \mu_{n}(d s) \rightarrow \int_{S} \omega(s) \mu_{0}(d s)$ as $n \rightarrow \infty$. Suppose that there exists $k>0$ such that $0 \leq g_{n}(s) \leq k \omega(s)$ for all $s \in S$ and $n \in \mathbb{N}$. Then, we have

$$
\int_{S} \limsup _{n \rightarrow \infty, s^{\prime} \rightarrow s} g_{n}\left(s^{\prime}\right) \mu_{0}(d s) \geq \limsup _{n \rightarrow \infty} \int_{S} g_{n}(s) \mu_{n}(d s)
$$

Proof Note that $h_{n}\left(s^{\prime}\right):=k \omega\left(s^{\prime}\right)-g_{n}\left(s^{\prime}\right) \geq 0$ for all $s^{\prime} \in S$ and $n \in \mathbb{N}$. By Lemma 3.2 in [36], we have

$$
\int_{S} \liminf _{n \rightarrow \infty, s^{\prime} \rightarrow s} h_{n}\left(s^{\prime}\right) \mu_{0}(d s) \leq \liminf _{n \rightarrow \infty} \int_{S} h_{n}(s) \mu_{n}(d s) .
$$

It is clear that

$$
\liminf _{n \rightarrow \infty, s^{\prime} \rightarrow s}\left(k \omega\left(s^{\prime}\right)-g_{n}\left(s^{\prime}\right)\right)=k \omega\left(s^{\prime}\right)-\limsup _{n \rightarrow \infty, s^{\prime} \rightarrow s} g_{n}\left(s^{\prime}\right) .
$$

Moreover, we have

$$
\liminf _{n \rightarrow \infty} \int_{S} h_{n}(s) \mu_{n}(d s)=\int_{S} k \omega\left(s^{\prime}\right) \mu_{0}\left(d s^{\prime}\right)-\limsup _{n \rightarrow \infty} \int_{S} g_{n}(s) \mu_{n}(d s) .
$$

Now observe that (12) follows easily from (13). 
Lemma 7 Assume that $c_{n} \stackrel{*}{\rightarrow} c_{0}$ in $F$. Then, for every $s^{\prime} \in S$, we have

$$
\limsup _{n \rightarrow \infty, z \rightarrow s^{\prime}} v\left(c_{n}(z)\right) \leq v\left(c\left(s^{\prime}\right)\right) \text {. }
$$

Proof Let $\left(z_{n}\right)$ be arbitrary sequence in $S$ converging to $s^{\prime}$. By Lemma 4 in [4], we obtain

$$
\limsup _{n \rightarrow \infty} v\left(c_{n}\left(z_{n}\right)\right) \leq v\left(c\left(s^{\prime}\right)\right) .
$$

Thus, (14) follows by taking supremum over all sequences $\left(z_{n}\right)$ converging to $s^{\prime}$ on the left-hand side of (15).

Let $V$ be the closed subset of all nonnegative functions $f$ in the Banach space $C(F \times S)$ such that $f(c, 0)=0$. Let $f \in V$ be fixed. For any $c \in F, s \in S$, define

$$
A_{o}(c)(s):=\arg \max _{i \in A(s)} u\left(s-i, W\left(\mathcal{E}_{i} v(c), \mathcal{E}_{i} f(c)\right)\right) \text { and } a_{o}(c)(s)=\min A_{o}(c)(s) .
$$

By Lemmas 3 and 4, the set $A_{o}(c)(s)$ is non-empty and compact and therefore, $a_{o}(c)(s)$ is well defined. Put for any $c \in F$

$$
(T f)(c, s):=\max _{i \in A(s)} u\left(s-i, W\left(\mathcal{E}_{i} v(c), \mathcal{E}_{i} f(c)\right)\right), \quad s \in S .
$$

\section{Lemma 8 T maps $V$ into itself.}

Proof Step 1. First note that from $q(\{0\} \mid 0)=1, u(0,0)=0$ and $W(0,0)=0$, it follows that $(T f)(c, 0)=0$. Assume that $c_{n} \stackrel{*}{\rightarrow} c_{0}$ in $F$ and $s_{n} \rightarrow s_{0}$ in $S$ as $n \rightarrow \infty$. For each $n \in \mathbb{N}$, choose any $i_{n} \in A_{o}\left(c_{n}\right)\left(s_{n}\right)$. Without loss of generality, it can be assumed that $i_{n} \rightarrow i_{0} \in A\left(s_{0}\right)$ as $n \rightarrow \infty$. By Lemma $3, \mathcal{E}_{i_{n}} f\left(c_{n}\right) \rightarrow \mathcal{E}_{i_{0}} f\left(c_{0}\right)$ as $n \rightarrow \infty$. From Lemmas 6 and 7, it follows that

$$
\limsup _{n \rightarrow \infty} \mathcal{E}_{i_{n}} v\left(c_{n}\right) \leq \int_{S} \limsup _{n \rightarrow \infty, s^{\prime} \rightarrow s} v\left(c_{n}\left(s^{\prime}\right)\right) q\left(d s \mid i_{0}\right) \leq \int_{S} v\left(c_{0}(s)\right) q\left(d s \mid i_{0}\right)=\mathcal{E}_{i_{0}} v\left(c_{0}\right) .
$$

Therefore, by our monotonicity and continuity assumptions on $u$ and $W$, we infer that

$$
\limsup _{n \rightarrow \infty}(T f)\left(c_{n}, s_{n}\right) \leq u\left(s_{0}-i_{0}, W\left(\mathcal{E}_{i_{0}} v\left(c_{0}\right), \mathcal{E}_{i_{0}} f\left(c_{0}\right)\right)\right) \leq(T f)\left(c_{0}, s_{0}\right) .
$$

Observe now that if $s_{0}=0$, then

$$
\liminf _{n \rightarrow \infty}(T f)\left(c_{n}, s_{n}\right) \geq(T f)\left(c_{0}, s_{0}\right)=(T f)\left(c_{0}, 0\right)=0 .
$$

Let $\mathcal{C}\left(c_{0}\right)$ be the set of all continuity points of the function $c_{0}$. Clearly, $0 \in \mathcal{C}\left(c_{0}\right)$ and $S \backslash \mathcal{C}\left(c_{0}\right)$ is countable. Assume that $s_{0}>0$ and define $D:=\left\{i \in\left[0, s_{0}\right): q\left(\mathcal{C}\left(c_{0}\right) \mid i\right)=1\right\}$. By (Q3), $D$ is a dense subset of $\left[0, s_{0}\right)$. Choose any $i \in D$. Then, $i \leq s_{n}$ for all but finitely many $n \in \mathbb{N}$. Under our conditions on $v$ and $\omega$, the sequence $\left(v\left(c_{n}(\cdot)\right)\right.$ satisfies the assumptions of the dominated convergence theorem and it converges to $v\left(c_{0}(\cdot)\right) q(\cdot \mid i)$-a.e. Therefore, we have that $\lim _{n \rightarrow \infty} \mathcal{E}_{i} v\left(c_{n}\right)=\mathcal{E}_{i} v\left(c_{0}\right)$. By Lemma 3, we also have $\lim _{n \rightarrow \infty} \mathcal{E}_{i} f\left(c_{n}\right)=\mathcal{E}_{i} f\left(c_{0}\right)$. Therefore,

$$
\begin{aligned}
\liminf _{n \rightarrow \infty}(T f)\left(c_{n}, s_{n}\right) & \geq \lim _{n \rightarrow \infty} u\left(s_{n}-i, W\left(\mathcal{E}_{i} v\left(c_{n}\right), \mathcal{E}_{i} f\left(c_{n}\right)\right)\right) \\
& =u\left(s_{0}-i, W\left(\mathcal{E}_{i} v\left(c_{0}\right), \mathcal{E}_{i} f\left(c_{0}\right)\right)\right) .
\end{aligned}
$$


Assume that $\tilde{i} \in\left[0, s_{0}\right) \backslash D$ or $\tilde{i}=s_{0}$. Then, $\tilde{i}>0$ and we can take $i \in D$ such that $i<\tilde{i}$ and $i \uparrow \tilde{i}$. Since $c_{0}$ is continuous from the left, by Lemma 5, the function $i \rightarrow \mathcal{E}_{i} v\left(c_{0}\right)$ is also continuous from the left. Thus, we infer that

$$
\lim _{i \uparrow \tilde{i}} u\left(s_{0}-i, W\left(\mathcal{E}_{i} v\left(c_{0}\right), \mathcal{E}_{i} f\left(c_{0}\right)\right)\right)=u\left(s_{0}-\tilde{i}, W\left(\mathcal{E}_{\tilde{i}} v\left(c_{0}\right), \mathcal{E}_{\tilde{i}} f\left(c_{0}\right)\right)\right) .
$$

This equality and (19) imply that

$$
\liminf _{n \rightarrow \infty}(T f)\left(c_{n}, s_{n}\right) \geq u\left(s_{0}-i, W\left(\mathcal{E}_{i} v\left(c_{0}\right), \mathcal{E}_{i} f\left(c_{0}\right)\right)\right)
$$

for all $i \in A\left(s_{0}\right)$. Hence,

$$
\liminf _{n \rightarrow \infty}(T f)\left(c_{n}, s_{n}\right) \geq(T f)\left(c_{0}, s_{0}\right) .
$$

From (18) and (20), it follows that $T f$ is continuous at any point $\left(c_{0}, s_{0}\right) \in F \times S$.

Step 2. By (Q5), we have $\int_{S} \omega\left(s^{\prime}\right) q\left(d s^{\prime} \mid i\right) \leq \kappa_{0} \omega(s)$ for all $s \in S, i=s-a \in A(s)$. Note that $i \leq s, v\left(c\left(s^{\prime}\right)\right) \leq v\left(s^{\prime}\right)$ for all $s^{\prime} \in S$ and

$$
\mathcal{E}_{i} v(c)=\int_{S} v\left(c\left(s^{\prime}\right)\right) q\left(d s^{\prime} \mid i\right) \leq \int_{S} \omega\left(s^{\prime}\right)\|v\|_{\omega} q\left(d s^{\prime} \mid i\right) \leq\|v\|_{\omega} \kappa_{0} \omega(s) .
$$

We also have

$$
\mathcal{E}_{i} f(c)=\int_{S} f\left(c, s^{\prime}\right) q\left(d s^{\prime} \mid i\right) \leq \int_{S} \omega\left(s^{\prime}\right)\|f\|_{\omega} q\left(d s^{\prime} \mid i\right) \leq\|f\|_{\omega} \kappa_{0} \omega(s) .
$$

From (U8) and (D), it follows that

$$
\begin{aligned}
u\left(s-i, W\left(\mathcal{E}_{i} v(c), \mathcal{E}_{i} f(c)\right)\right) & \leq u\left(s-i, W\left(\mathcal{E}_{i} v(c), 0\right)\right)+\delta\left(\mathcal{E}_{i} f(c)\right) \\
& \leq u\left(s-i, W\left(\mathcal{E}_{i} v(c), 0\right)\right)+\mathcal{E}_{i} f(c) .
\end{aligned}
$$

From (21)-(23), we conclude that

$$
u\left(s-i, W\left(\mathcal{E}_{i} v(c), \mathcal{E}_{i} f(c)\right)\right) \leq u\left(s, W\left(\|v\|_{\omega} \kappa_{0} \omega(s), 0\right)\right)+\|f\|_{\omega} \kappa_{0} \omega(s) .
$$

By (24) and (U7), we obtain that

$$
u\left(s-i, W\left(\mathcal{E}_{i} v(c), \mathcal{E}_{i} f(c)\right) \leq K \omega(s)\right.
$$

for all $s \in S$, and for $K=\kappa_{1}+\|f\|_{\omega} \kappa_{0}$. Thus, we have $0 \leq(T f)(c, s) \leq K \omega(s)$ for all $s \in S$ and consequently $\|T f\|_{\omega} \leq K<\infty$.

Lemma 9 For every $f_{1}, f_{2} \in V$, we have

$$
\left\|T f_{1}-T f_{2}\right\|_{\omega} \leq \delta\left(\kappa_{0}\left\|f_{1}-f_{2}\right\|_{\omega}\right) .
$$

Proof By (U8), for any $f_{1}, f_{2} \in V$, we have

$$
\begin{aligned}
\left|\left(T f_{1}\right)(c, s)-\left(T f_{2}\right)(c, s)\right| & \leq \delta\left(\sup _{i \in A(s)} \int_{S}\left|f_{1}\left(c, s^{\prime}\right)-f_{2}\left(c, s^{\prime}\right)\right| q\left(d s^{\prime} \mid i\right) \mid\right) \\
& \leq \delta\left(\sup _{i \in A(s)} \int_{S} \omega\left(s^{\prime}\right)\left\|f_{1}-f_{2}\right\|_{\omega} q\left(d s^{\prime} \mid i\right)\right) .
\end{aligned}
$$

This fact, (1) and (Q5) imply that

$$
\left|\left(T f_{1}\right)(c, s)-\left(T f_{2}\right)(c, s)\right| \leq \delta\left(\kappa_{0}\left\|f_{1}-f_{2}\right\|_{\omega}\right) \omega(s), \quad s \in S, c \in F .
$$

Hence, (25) follows. 
Proof of Theorem 1 If $\kappa_{0} \leq 1$, then, by Lemma $9, T$ is a $\delta$-contraction mapping from $V$ into itself. If $\kappa_{0}>1$, then we define $\delta_{0}(r)=\delta\left(\kappa_{0} r\right)$. By (D) and (25), $T$ is a $\delta_{0}$-contraction mapping. By Proposition 1, there exists a unique $U^{*} \in V$ such that $T U^{*}=U^{*}$.

Let

$$
A_{o}^{*}(c)(s):=\arg \max _{i \in A(s)} u\left(s-i, W\left(\mathcal{E}_{i} v(c), \mathcal{E}_{i} U^{*}(c)\right)\right), \quad c \in F, \quad s \in S .
$$

The set $A_{o}^{*}(c)(s)$ is non-empty and compact. Define the mapping $a_{o}^{*}(c)(s):=\min A_{o}^{*}(c)(s)$, $s \in S$. By Proposition 2, $a_{o}^{*}: F \rightarrow I$. Hence, $a_{o}^{*}(c)$ is continuous from the left. We show that $a_{o}^{*}$ is continuous when both $F$ and $I$ are given the topology of weak convergence. Assume that $c_{n} \stackrel{*}{\rightarrow} c_{0}$ in $F$ as $n \rightarrow \infty$. Put $y_{n}^{*}:=a_{o}^{*}\left(c_{n}\right)$, Let $y_{o}^{*}$ be any accumulation point of the sequence $\left(y_{n}^{*}\right)$ in the compact space $I$. We need to show that $y_{o}^{*}=a_{o}^{*}\left(c_{0}\right)$. Clearly, $y_{o}^{*}(0)=a_{o}^{*}\left(c_{0}\right)(0)=0$. Let $\left(n^{\prime}\right)$ be a subsequence of positive integers such that $y_{n^{\prime}}^{*} \stackrel{*}{\rightarrow} y_{0}^{*}$ as $n^{\prime} \rightarrow \infty$. Let $\mathcal{C}\left(y_{0}^{*}\right)$ be the set of all continuity points of the function $y_{0}^{*}$. Since $y_{0}^{*} \in I, S \backslash \mathcal{C}\left(y_{0}^{*}\right)$ is countable, the set $\mathcal{C}\left(y_{0}^{*}\right)$ is dense in $S$. Choose any $s \in \mathcal{C}\left(y_{0}^{*}\right)$. From Step 1 of the proof of Lemma 8 (with $i_{n}=y_{n^{\prime}}^{*}(s), i_{0}=y_{0}^{*}(s)$ and $s_{n}=s$ ), we can conclude that $y_{0}^{*}(s) \in A_{o}^{*}\left(c_{0}\right)(s)$, for any $s \in \mathcal{C}\left(y_{0}^{*}\right)$. Since $y_{0}^{*}$ is continuous at $s$, by Lemma $2, A_{o}^{*}\left(c_{0}\right)(s)$ is a singleton. Therefore, $y^{*}(s)=a_{o}^{*}\left(c_{0}\right)(s)$ for all $s \in \mathcal{C}\left(y_{0}^{*}\right)$. As both functions $y_{0}^{*}$ and $c_{0}$ are left continuous on $S$ and $\mathcal{C}\left(y_{0}^{*}\right)$ is dense in $S$, we conclude that $y_{0}^{*}(s)=a_{o}^{*}\left(c_{0}\right)(s)$ for all $s \in S$. Thus, we have shown that the mapping $a_{o}^{*}: F \rightarrow I$ is continuous. Let $\Psi(c)(s):=s-a_{o}^{*}(c)(s), s \in S, c \in F$. Obviously, $\Psi: F \rightarrow F$ is also a continuous mapping. Moreover, we know from Lemma 1 that $F$ is a compact convex subset of a locally convex topological vector space. By the Schauder-Tychonoff fixed-point theorem (see Aliprantis and Border [1] or Dugundji and Granas [16]), there exists some $c^{*} \in F$ such that $\Psi\left(c^{*}\right)=c^{*}$. Clearly, $\left(c^{*}, U^{*}\right)$ is an SMPE.

Remark 9 We have shown that $U^{*}$ is a fixed point of the mapping $T$ defined in (17). By Proposition $1, U^{*}$ is unique. Moreover, if $f_{0} \equiv 0$ and $f_{0} \in V$, then $\lim _{n \rightarrow \infty}\left\|T^{n} f_{0}-U^{*}\right\|_{\omega}=$ 0 .

In the remaining part of this section, we consider only assumptions of Theorem 2. By replacing the function $\omega$ with $v$ in Lemmas 4 and 5 and using condition (U5), one can prove that the mapping

$$
i \rightarrow \int_{S} v\left(c\left(s^{\prime}\right)\right) q\left(d s^{\prime} \mid i\right)
$$

is upper semicontinuous continuous from the left. Let

$$
\tilde{A}_{o}^{*}(c)(s):=\arg \max _{i \in A(s)} u\left(s-i, \mathcal{E}_{i} v(c)\right) \text { and } \tilde{a}_{o}^{*}(c)(s):=\min \tilde{A}_{o}^{*}(c)(s), \quad s \in S, c \in F .
$$

Proof of Theorem 2 A simple adaptation of the arguments used in the proof of Theorem 1 yields that the mapping $\tilde{a}_{o}^{*}: F \rightarrow I$ is continuous in the weak topology on $F$ and $I$. Therefore, the mapping $\tilde{\Psi}(c)(s):=s-\tilde{a}_{o}^{*}(c)(s)$ from $F$ into $F$ is continuous as well. By the Schauder-Tychonoff fixed-point theorem, there exists some $c^{*} \in F$ such that $\tilde{\Psi}\left(c^{*}\right)=c^{*}$. Clearly, $c^{*}$ is an SMPE.

Acknowledgements We thank Kevin Reffett and Łukasz Woźny for very stimulating discussions. The authors acknowledge the financial support from the National Science Centre, Poland: Grants 2016/23/B/HS4/02398 (Ł. Balbus) and 2016/23/B/ST1/00425 (A. Jaśkiewicz and A.S. Nowak). 
Open Access This article is distributed under the terms of the Creative Commons Attribution 4.0 International License (http://creativecommons.org/licenses/by/4.0/), which permits unrestricted use, distribution, and reproduction in any medium, provided you give appropriate credit to the original author(s) and the source, provide a link to the Creative Commons license, and indicate if changes were made.

\section{References}

1. Aliprantis C, Border K (2006) Infinite dimensional analysis: a hitchhiker's guide. Springer, New York

2. Balbus $€$ (2018) Markov perfect equilibria in OLG models with risk sensitive agents. Top Meth Nonlinear Anal. https://doi.org/10.12775/TMNA.2019.016

3. Balbus Ł, Jaśkiewicz A, Nowak AS (2015a) Bequest games with unbounded utility functions. J Math Anal Appl 427:515-524

4. Balbus Ł, Jaśkiewicz A, Nowak AS (2015b) Stochastic bequest games. Games Econ Behav 90:247-256

5. Balbus $€$, Jaśkiewicz A, Nowak AS (2015c) Existence of stationary Markov perfect equilibria in stochastic altruistic growth economies. J Optim Theory Appl 165:295-315

6. Balbus Ł, Jaśkiewicz A, Nowak AS (2016) Non-paternalistic intergenerational altruism revisited. J Math Econ 63:27-33

7. Balbus Ł, Jaśkiewicz A, Nowak AS (2018) Markov perfect equilibria in a dynamic decision model with quasi-hyperbolic discounting. Ann Oper Res (in press)

8. Balbus Ł, Reffett K, Woźny Ł (2013) A constructive geometrical approach to the uniqueness of Markov stationary equilibrium in stochastic games of intergenerational altruism. J Econ Dyn Control 37:10191039

9. Balbus Ł, Reffett K, Woźny Ł (2018a) Dynamic games in macroeconomics. In: Başar T, Zaccour G (eds) Handbook of dynamic game theory, vol 2. Springer, Cham, pp 729-778

10. Barro R (1974) Are government bonds net wealth? J Polit Econ 82:1095-1118

11. Bernheim D, Ray D (1983) Altruistic growth economies I. Existence of bequest equilibria. Technical report no 419, Institute for Mathematical Studies in the Social Sciences, Stanford University

12. Bertsekas DP, Shreve SE (1978) Stochastic optimal control: the discrete time case. Academic Press, New York

13. Billingsley P (1968) Convergence of probability measures. Wiley, New York

14. Boyd JH III (1990) Recursive utility and the Ramsey problem. J Econ Theory 50:326-345

15. Becker R, Boyd JH (1997) Capital theory equilibrum analysis and recursive utility. Wiley, New York

16. Dugundji J, Granas A (2003) Fixed point theory. Springer, New York

17. Durán J (2000) On dynamic programming with unbounded returns. Econ Theory 15:339-352

18. Durán J (2003) Discounting long run average growth in stochastic dynamic programs. Econ Theory 22:395-413

19. Dutta PK, Sundaram R (1992) Markovian equilibrium in a class of stochastic games: existence theorems for discounted and undiscounted models. Econ Theory 2:197-214

20. Epstein LG, Zin SE (1989) Substitution, risk aversion, and the temporal behavior consumption and asset returns: a theoretical framework. Econometrica 57:937-969

21. Galperti S, Strulovici B (2017) A theory of intergenerational altruism. Econometrica 85:1175-1218

22. Hernández-Lerma O, Lasserre JB (1999) Further topics on discrete-time Markov control processes. Springer, New York

23. Hori H (1997) Dynamic allocation in an altruistic overlapping generations economy. J Econ Theory 73:292-315

24. Jaśkiewicz A, Nowak AS (2011) Stochastic games with unbounded payoffs: applications to robust control in economics. Dyn Games Appl 1:253-279

25. Jaśkiewicz A, Nowak AS (2018a) Non-zero-sum stochastic games. In: Başar T, Zaccour G (eds) Handbook of dynamic game theory, vol 1. Springer, Cham, pp 281-344

26. Jaśkiewicz A, Nowak AS (2018b) On symmetric stochastic games of resource extraction with weakly continuous transitions. TOP 26:239-256

27. Jaśkiewicz A, Matkowski J, Nowak AS (2014) Generalised discounting in dynamic programming with unbounded returns. Oper Res Lett 42:231-233

28. Leininger W (1986) The existence of perfect equilibria in model of growth with altruism between generations. Rev Econ Stud 53:349-368

29. Loury G (1981) Intergenerational transfers and the distribution of earnings. Econometrica 49:843-867

30. Maliar L, Maliar S (2016) Ruling out multiplicity of smooth equilibria in dynamic games: a hyperbolic discounting example. Dyn Games Appl 6:243-261 
31. Matkowski J (1975) Integral solutions of functional equations. Dissertationes Mathematicae 127:1-68

32. Milgrom P, Shannon C (1994) Monotone comparative statics. Econometrica 62:157-180

33. Pearce DG (2008) Nonpatemalistic sympathy and the inefficiency of consistent intertemporal plans. In: Jackson MO, McLennan A (eds) Foundations of Microeconomic Theory, A volume in honor of Hugo F. Sonnenschein. Springer, Berlin, pp 215-231

34. Phelps E, Pollak R (1968) On second best national savings and game equilibrium growth. Rev Econ Stud 35:195-199

35. Ray D (1987) Nonpaternalistic intergenerational altruism. J Econ Theory 40:112-132

36. Serfozo R (1982) Convergence of Lebesgue integrals with varying measures. Sankhya Indian J Statist 44:380-402

37. Sundaram RK (1989) Perfect equilibrium in a class of symmetric dynamic games. J Econ Theory 47:153177 (Corrigendum: J Econ Theory 49:385-187)

38. Topkis D (1978) Minimizing a submodular function on a lattice. Oper Res 26:305-321

39. Topkis D (1998) Supermodularity and complementarity. Princeton University Press, New Jersey

40. Wessels J (1977) Markov programming by successive approximations with respect to weighted supremum norms. J Math Anal Appl 58:326-335

Publisher's Note Springer Nature remains neutral with regard to jurisdictional claims in published maps and institutional affiliations. 